\title{
Collaborative Knowledge Construction through Shared Representations
}

\author{
Daniel D. Suthers \\ University of Hawai $i$ \\ suthers@hawaii.edu
}

\begin{abstract}
This paper is concerned with the question of how activity mediated by shared representations-notations that are manipulated by more than one person during a collaborative task-might constitute knowledge construction activity. The paper begins with a brief review of theoretical perspectives on how representations mediate collaborative knowledge construction, to identify the kinds of events we would look for as evidence of knowledge construction in via a representational medium. Then the paper draws on data from a prior study in which participants collaborated via a graphical representation as well as a verbal "chat" tool, to identify instances of such events and illustrate ways in which the activity of two individuals can be coupled and joined into a larger cognitive (and sometimes knowledge construction) activity distributed across the persons and representations they are manipulating.
\end{abstract}

\section{Introduction}

I am studying how software tools that support learners' construction of knowledge representations (e.g., concept maps, evidence maps, evidence tables) are used by collaborating learners, and consequently how to design such tools to more effectively support collaboration. The value and role of external representations (inscriptions) in mediating collaborative inquiry has been demonstrated by [12] and others, including some of the present author's own work [17]. These studies have examined face-to-face collaborations. Given the importance of co-present cues for grounding communication [3] and other issues with interaction at a distance [11], we might expect that shared representations have reduced utility online; but the possibility has also been raised that computer media can go "beyond being there" in enabling new forms of interaction [8].

In a previous study [16], I found that online collaborators treated a graphical representation of evidence as a medium through which collaboration took place as well as its object, proposing new ideas by entering them directly in the graph before engaging in (usually brief) confirmation dialogues in a textual chat tool. In contrast to face-to-face users of shared representations, gesture was almost never used online and verbal deixis almost always referenced temporally recent information; instead, direct manipulation of the graph was used to reintroduce old information. In general, actions in the graph appeared to be an important part of participants' conversations with each other, and in fact was at times the sole means of interaction. It was clear that all mutable media become part of the communication medium in virtual interaction, and therefore should be designed together with this use in mind.

These observations led to the questions of the quality of interactions through the graph. Are participants merely operating in parallel on the same workspace, or are they in some sense having a conversation through the graph? If so, is this conversation at all significant from a learning standpoint: can true knowledge construction take place through shared representations?

To answer these questions, I identified interactions from our corpus that appeared to constitute collaboration through the nonverbal as well as verbal media, and am engaged in a qualitative analysis of these examples. The purpose of this analysis is to understand how participants made use of the graph representation to mediate meaning making activity, by examining how participants use actions on the representations to build on each others' ideas. The larger goal is to identify affordances of shared representations for online collaboration and their implications for the design of representational support for collaborative knowledge construction.

For this case study, I drew upon three sessions (of 10) that were promising due to their interactive use of the graph and varying uses of the chat media. I am conducting an analysis in a bottom-up manner, working from literal actions first to a referential level of analysis and then to an intentional level, in a manner similar to [10]. Initially my colleagues and I coded the literal actions taken by participants in the shared workspace. Then my student Ravikiran Vatrapu and I identified ways in which information "flows" between participants through the graph, as evidenced by their references to information in the graph. I am now layering on top of this analysis my own interpretations of the intentions behind these references, and seeking evidence of knowledge construction activity. 


\subsection{What is collaborative knowledge construction, that representations might support it?}

Stahl [14] provides a detailed model of knowledge construction processes, a model that includes more than several cognitive and social processes. (Stahl's term is "knowledge building," which is not identical to "knowledge construction," but the distinction need not be taken up here: see [13] and earlier writings by these authors.) While Stahl's model is useful for understanding the richness of knowledge building interactions, I need more succinct criteria for whether knowledge construction is taking place. I am currently operating under the working definition that knowledge construction is evidenced by the accretion of interpretations on an information base that is simultaneously expanded by information seeking and transformations. The act of interpretation may take the form of explicit sense-making commentary, but it may also take place through the transformation and integration of representations of the information base. Then, collaborative knowledge construction takes place when multiple participants contribute to this accretion of interpretations by building, commenting on, transforming and integrating a shared information base.

What then is the role of external representations in knowledge construction? Clearly, external representations can serve as the medium in which information is accumulated, transformed and interpreted. Yet, if we can be more specific about how representations support the collaborative aspects of knowledge construction then we can have a better idea of what to look for in an analysis. Several theoretical perspectives on learning are briefly reviewed here to appreciate their insights into how external representations might serve as resources for collaborative sense-making, and to identify implications for analysis.

Let us look first to an influential theory of linguistic communication. Participants' actions in representational media (as well as language) can be understood in terms of Clark's model of grounding [3, 9]. We can restate grounding in terms of actions on a nonlinguistic (or semilinguistic) representation as follows: a participant expresses an idea in the representation; another participant acts on that representation in a manner that provides evidence of understanding the first participant's intent in a certain way; the first participant can choose to accept this action as evidence of sufficient understanding, or, if the evidence is insufficient, initiate repair. Under the grounding perspective, the analyst would look for sequences of actions in which one participant's action on a representation is taken up by another participant in a manner that indicates understanding of its meaning, and the first participant signals acceptance.

A problem for analysis is that this final signal of acceptance is often implicit, so can be difficult to identify. For example, it can consist merely of continuing the interaction rather than initiating repair of a breakdown. Also, an analysis based solely on grounding theory will not tell us much more than when participants have understood each other or have identified a need to repair a misunderstanding. It does not tell us whether participants are accumulating interpretations of a growing knowledge base. However, this perspective does suggest that we might view interaction through representations as a form of nonverbal or semi-verbal conversation.

Socio-cognitive conflict theory [5] and cognitive dissonance theory [6] suggest various ways in which learning can result from social interaction in which individuals encounter ideas that are different from their own. The individual is challenged to reconsider his or her beliefs, potentially leading to change, or to explain and justify those beliefs to others, leading to clarifications and elaborations that might not have otherwise taken place. These clarifications and elaborations may be seen as acts of interpretation. Representations that externalize one's beliefs can make beliefs explicit enough for one's interlocutors to notice conflicts, thereby initiating a sociocognitive process of learning. This noticing is especially likely to occur if multiple participants have externalized their beliefs in a representational system that makes conflicts explicit, and processes of elaboration and reconceptualization may also be externalized in interactions between participants that take place via shared representations. Under the socio-cognitive conflict perspective, we would want to identify situations in which the externalization of ideas led to identification of differences of interpretation that were subsequently taken up by at least one of the individuals involved. In addition to overt verbal argumentation, clues that conflict is being addressed might include revision or deletion of the others' ideas or the use of an explicit conflict relation between one's own and others' ideas, if the representation provides for such relations.

The foregoing perspective is useful as far as it goes, but limiting in that it treats participants as separate cognitive entities that interact via language and (other) notations, yet retains the locale of knowledge construction activity within the individual. A distributed cognition perspective [7] suggests that cognitive activities such as knowledge construction are distributed across individuals and information artifacts through and with which they interact. In this perspective, the informationtransformative and interpretive components of a cognitive activity can occur across multiple individuals via external representations. An individual can perform a cognitive act that results in creation of or a change to a representation 
that is shared with another individual, who subsequently takes up this information and adds to, transforms or interprets it in a new way, again resulting in a change to the representation that may be taken up by the first individual, and so on. Knowledge construction, being a form of cognition, can also take place with and through external representations of various (visual and symbolic) forms not limited to language. Therefore, under the distributed cognition perspective we would look for transformations of representations across individuals where those transformations can be interpreted as an intersubjective cognitive process such as knowledge construction. Examples include merging, revising, and connecting representations of ideas.

Another perspective I considered is the activity theoretic perspective [1]. This school of thought presents a rich collection of claims and insights about the situated nature of activity. It considers how activity is situated in a larger context that includes not only the self, the object or topic of interest, and tools such as the external representations with which we are concerned, but also one's community, one's role in this community, and the norms for behavior in the community. This breadth of analysis does not apply to the activity analyzed in this paper, an artificial laboratory study that lacks embedding in a community. However, there is one concept that is valuable for understanding even artificially initiated episodes of collaboration with artifacts: the concept of mediation. When we examine the relationship between any two elements of an activity system (the subject, object, tool, community, roles, rules), we can sometimes benefit from asking how any third element mediates the relationship between the first two. For example, we have seen from the discussion of distributed cognition that representations mediate between individuals. I interpret mediation to mean not only a channel through which the relationship is formed, but also something that influences the form the relationship takes. For example, rules influence as well as mediate one's relationship to a community. Similarly, external representations mediate collaborative inquiry when collaborators try to make sense of them [12]. They can also influence one's interactions with others by crystallizing prior practice and suggesting specific epistemic activities [4] or facilitating or inhibiting cognitive activity [2]. Under an activitytheoretic perspective, we would analyze collaborative use of representations by looking for ways in which the representation mediates (makes possible and guides) interactions between participants by virtue of its form. This viewpoint is certainly consistent with the distributed cognition perspective, as well as my own perspective discussed below, which makes the notion of "guides ... by virtue of its form" more specific.

In my own prior work [17], I identified three roles of external representations that are unique to situations in which a group is constructing and manipulating shared representations as part of a constructive activity, two of which suggest events to look for in an analysis.

1. Initiating and capturing the results of negotiations of meaning. An individual who wishes to add to or modify a shared representation may feel some obligation to obtain agreement from one's group members, leading to negotiations about and justifications of representational acts. This discourse will include negotiations of meaning and shared belief that would not be necessary in the individual case, where one can simply change the representation as one wishes. The creative acts afforded by a given representational notation may affect which negotiations of meaning and belief take place. An implication for analysis is that we should look for interactions across representational and linguistic media, for example identifying discussions initiated as participants prepare to act upon a representation.

2. Supporting conversations through deixis. The components of a collaboratively constructed representation, having arisen from negotiations of the type just discussed, evoke in the minds of the participants meanings beyond that which external observers might be able to discern by inspection of the representations alone. These components can serve as an easy way to refer to ideas previously developed, this reference being accomplished by deixis (reference) rather than specific verbal descriptions. In this manner, collaboratively constructed external representations facilitate subsequent negotiations, increasing the conceptual complexity that can be handled in group interactions and facilitating elaboration on previously represented information. An implication for analysis is that we should identify ways in which participants use representations as a means of referring to ideas. As discovered in [16], online such references will likely take the form of direct manipulations of the representations rather than verbal or gestural deixis.

With this background, we will now focus on the specifics of the analysis undertaken for the present paper, an analysis that is producing examples of the kinds of interactions that theory tells us we should look for.

\section{Method}

The data that is the object of the current analysis was taken from a previous experimental study, reported in [16]. I begin by briefly describing how the sessions were conducted before describing the analysis undertaken in the present study. 


\subsection{Participants and Procedure}

In all of the studies leading up to and including the present study, participants consisted of self-selected, same-gender pairs from introductory natural science courses at the University of Hawai' i.

The participants' task was to propose and evaluate hypotheses concerning the cause of ALS-PD, a neurological disease with an unusually high occurrence on Guam that has been studied by the medical community for over 50 years without resolution. (A recent hypothesis concerning consumption of fruit eating bats as a vector for transmission of a neurotoxin to humans is promising.)

The experimental software (see figure 1) provided a graphical tool for constructing representations of the data, hypotheses, and evidential relations that participants gleaned from the information pages. The graph tool was based on version 3 of Belvedere [15], and enabled one to build a graph of nodes (data items and hypotheses) and links (evidential relations) representing an evidence model. Links can be created to represent consistency $(+)$, inconsistency (-) or unspecified (?) relations. (Belvedere is being used for historical reasons -- its availability to the investigator -- is is not my purpose to defend its particular design here. It provides a workable representational tool for the kinds of tasks we posed to participants, and ample representational flexibility for us to discover how people use representations without the complexities of a less constrained or more sophisticated tool.)

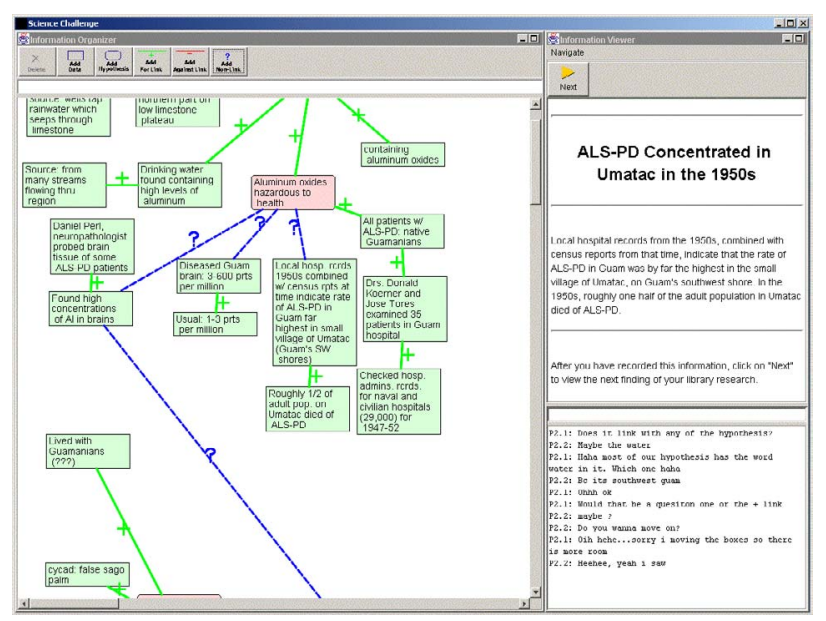

Figure 1. Experimental interface

An information window enabled participants to advance through a series of textual pages presenting information on ALS-PD. The sequence was designed such that later pages sometimes affected the interpretation of information seen several pages earlier, making the use of an external memory important.
In the study on which this analysis derives its data [16], the software was modified for synchronous online collaboration with the addition of a chat tool. Messages typed into an entry box were sent to both participants' shared chat displays once the Return key was pressed. Also, the software supported gestural deixis in two ways; one being automatic and the other requiring more deliberate action on the part of the user. If the user passed the cursor over an object, the fill-color of the object changed to blue. This was intended to enhance the deictic value of the cursor by making its location more visible. If the user deliberately selected an object with the cursor, the object was highlighted in yellow. The online version of the software replicated both of these color changes to the remote display. To maximize the potential for online participants to use this option for gestural deixis, we demonstrated this highlighting to them.

The pairs were given a 10-minute introduction to the problem, the task and the software. One participant was then led to a separate computer in a different room. They then engaged in a 12-minute warm-up exercise on an unrelated problem (mass extinctions). The main problem consisted of 15 informational pages on the ALS-PD disease, and participants were allowed to continue their interaction until they felt they had reached a conclusion. At the conclusion of the session, participants were given additional tasks not relevant for the present paper (see $[16,17])$.

Transcripts of chat messages and user actions in the graph were automatically logged in the online sessions. In the prior study, our analysis was concerned with how participants used the external representation as a resource for conversation, particularly by leveraging its potential for deictic reference to previously represented ideas and potentially new relationships between them. The present study is based on the same data, but focuses case examples of how knowledge construction is accomplished via manipulations of representations.

\subsection{Analysis}

Initially, analyses were attempted using linear transcripts. However, it was difficult to "see" how participants were interacting with each other in this format. Therefore, we began to explore other formats, eventually settling on a mixed tabular/graphical representation of the sessions (see figures 3 and 5). These represented the activity (chat and changes to the representation) of Participant 1 (P1) in the left hand column, and activity of Participant 2 (P2) in the right hand column. An empty column was left in the middle for annotations indicating "information uptake" relations between actions. We used a diagrammatic notation for information update. An arrow is drawn from action A1 to action A2 if A2 builds on the information in A1. 
Examples include editing or linking to prior information, or cross-modal references such as a chat comment about an item in the graph. The arrow is directed from past to future, as it shows the "flow" of information between past and future actors (which may be the same or different participants) via the representation. The links had to meet the criteria that the uptake identified is plausibly based on the informational content or attitude towards that information of the uptaken act or representation. There must be evidence that the uptaker is responding to one of these. (For example, merely moving things around is not counted.)

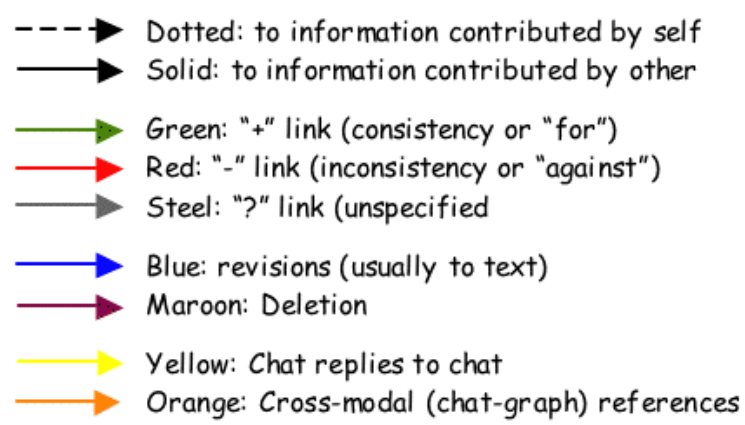

Figure 2. Key for uptake relations

The uptake instances were categorized in a manner reflected in the key of figure 2. As we are interested in collaboration through the representations rather than just individual use of the representations, we encoded this distinction: Dashed lines represent manipulation of items that were most recently manipulated by the same participant, while solid lines represent manipulations of items that were most recently manipulated by the other participant. Therefore, solid lines represent potential collaborative knowledge construction in the sense of informational or attitudinal uptake from one actor to another. This coding is based on the actor who most recently manipulated an item in the persistent representation, not necessarily the originator of the item, because we wanted to capture the "back and forth" of comanipulation of a representation. If references only went back to the original creation of an information item then it would not be possible to trace out dialectic interaction. Under this coding, one can identify coarse patterns of interaction as follows:

Vertical lines indicate that participants are revisiting prior information. Revisited items are almost always information that is expressed in the persistent representation (the graph). Therefore, an abundance of long vertical lines indicate that the representation is doing its job of preserving and allowing its users to return to previously encountered or expressed information.

Solid horizontal lines indicate that there is information exchange, and therefore potentially knowledge construction, between participants. (Solid lines always have a horizontal component, because they indicate information flow between participants, who's actions are represented in separate columns.)

Color indicates the relationship between the items taken up. For example, red and green indicate that an inconsistency or consistency relation (respectively) is being noted. Therefore presence of these colors suggests the nature of the argumentation. Blue is used for revisions, and maroon for deletions. Red and maroon suggest that there may be conflict; green and blue suggest the accretion and refinement of ideas.

We now turn to case examples of how collaborative knowledge construction can be accomplished via shared representations. The examples are presented in two notations: the referential analysis just described, and a tabular representation. The tabular representation has the advantages that time stamps are provided, and it is easier to read as a single sequence of actions. The time stamps are important for determining whether participants are working simultaneously or have had time to see and react to the previous action. For instance, in the forthcoming example (figure 3) we cannot rule out the possibility that the link D26+*D05 added at 15:51:08 was undertaken simultaneously with the deletion of D27 at 15:51:09 as far as the participants were concerned. Table 1 provides codes used in the tabular examples.

\section{Table 1. Codes used in examples}

\begin{tabular}{lll}
\hline Acts: & A & Object added to representation \\
& C & Chat \\
& D & Object deleted from representation \\
& G & Gesture on the indicated objects \\
M & Object modified in representation \\
& S & Spoken content \\
\hline Objects: & $*$ & Previously represented object is being \\
& & reintroduced into the conversation \\
& D & Data object \\
H & Hypothesis object
\end{tabular}

\section{Case Examples}

In this section I present examples of interaction through both the graph and the chat facility, and my interpretation of them. 


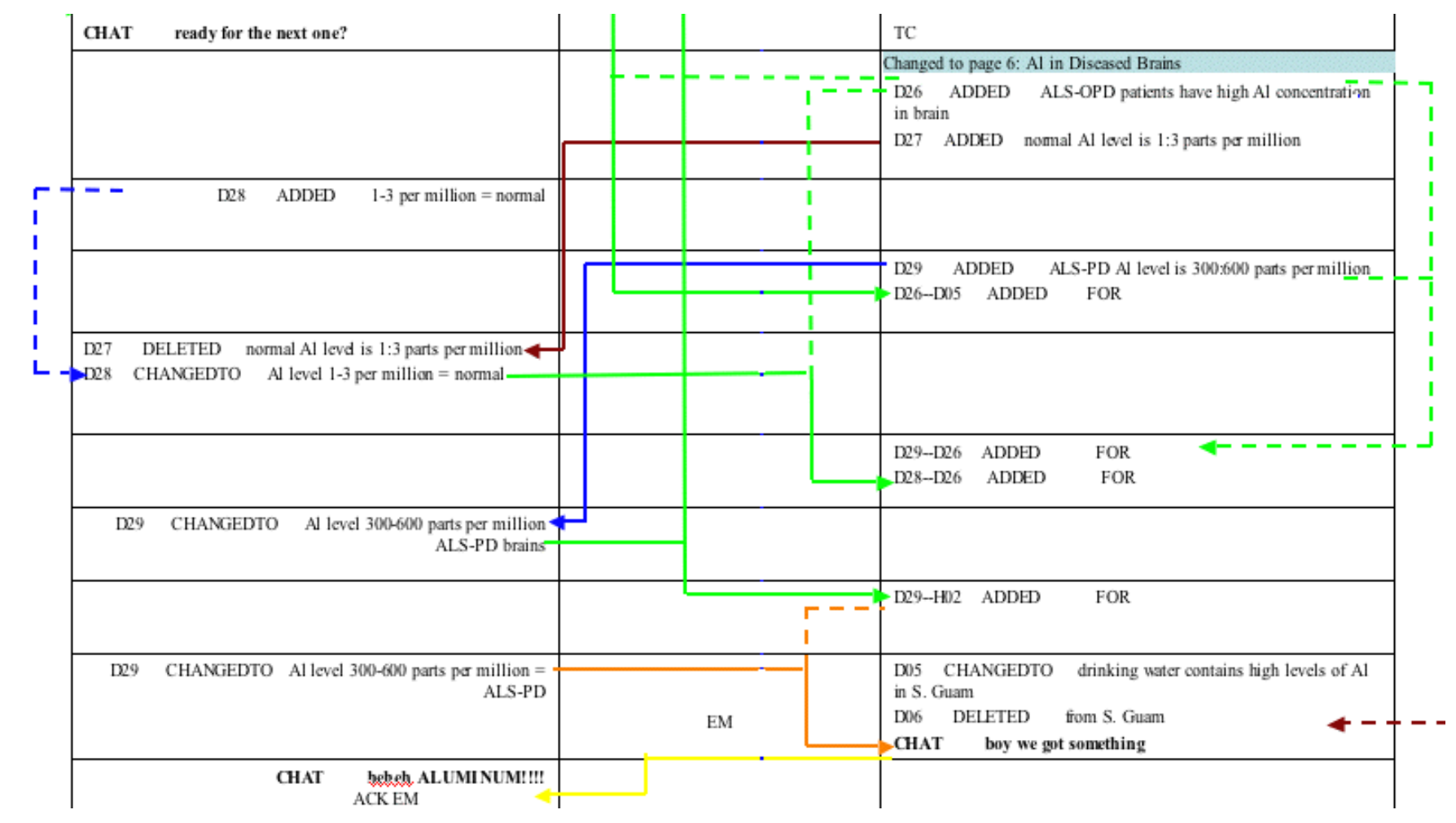

\begin{tabular}{lll}
\hline Previous Objects & $\mathrm{H} 02$ & $\mathrm{Al}$ or AlO is the cause \\
& $\mathrm{D} 05$ & drinking water contains high levels of A1 \\
& D06 & from S. Guam \\
\hline
\end{tabular}

Context: Participants have just read page titled "High Concentrations of Aluminum Found in Diseased Brains" which states:

"Neuropathologist Daniel Perl X-ray probed the brain tissue of some ALS-PD patients. He found unusually high concentrations of aluminum in those brains. He says, "Normally, the background level of aluminum in a neuron is from one to three parts per million. In the diseased Guam brains we're getting from three hundred to six hundred parts per million." "'

\begin{tabular}{|c|c|c|c|c|}
\hline Time & Who & Act & Object(s) & Chat or graph content [spelling as given] \\
\hline $15: 49: 51$ & \multirow[t]{2}{*}{$\mathrm{P} 2$} & $\mathrm{~A}$ & D26 & ALS-PD patients have high Al concentration in brain \\
\hline $15: 50: 20$ & & A & D27 & normal Al level is $1: 3$ parts per million \\
\hline $15: 50: 35$ & P1 & A & D28 & $1-3$ per million $=$ normal \\
\hline $15: 50: 51$ & \multirow[t]{2}{*}{$\mathrm{P} 2$} & A & $\mathrm{D} 29$ & ALS-PD Al level is 300:600 parts per million \\
\hline $15: 51: 08$ & & A & D26+*D05 & \\
\hline 15:51:09 & \multirow[t]{2}{*}{ P1 } & $\mathrm{D}$ & $\mathrm{D} 27$ & {$[$ deleted] $\ldots$} \\
\hline $15: 51: 26$ & & $\mathrm{M}$ & D28 & Al level $1-3$ per million = normal \\
\hline $15: 52: 21$ & \multirow[t]{2}{*}{$\mathrm{P} 2$} & $\mathrm{~A}$ & $\mathrm{D} 29+\mathrm{D} 26$ & \\
\hline $15: 52: 24$ & & A & $\mathrm{D} 28+\mathrm{D} 26$ & \\
\hline $15: 52: 31$ & P1 & $\mathrm{M}$ & D29 & Al level 300-600 parts per million ALS-PD brains \\
\hline $15: 52: 47$ & $\mathrm{P} 2$ & A & $\mathrm{D} 29+* \mathrm{H} 02$ & \\
\hline $15: 52: 52$ & P1 & $\mathrm{M}$ & D29 & Al level 300-600 parts per million $=$ ALS-PD \\
\hline $15: 53: 25$ & P2 & $\mathrm{M}$ & D05 & drinking water contains high levels of $\mathrm{Al}$ in $\mathrm{S}$. Guam \\
\hline \multirow[t]{2}{*}{$15: 53: 29$} & & $\mathrm{D}$ & D06 & from S. Guam \\
\hline & & M & [various] & [repositions various objects for 44 seconds] \\
\hline $15: 54: 13$ & & $\mathrm{C}$ & & boy we got something \\
\hline 15:54:39 & $\mathrm{P} 1$ & $\mathrm{C}$ & & heheh ALUMINUM!!!! \\
\hline
\end{tabular}

Figure 3. Example 1: Collaboration through the graph 


\subsection{Example 1: Collaborating through the graph}

The first example (figures 3 and 4) provides a basic example of collaboration through the graph leading to a conclusion that is acknowledged verbally. The detailed explanation provided for this example will also serve to familiarize the reader with the notations.

The participants had previously represented a hypothesis (H02) that aluminum is the cause of the disease, and two data items, D05 and D06, these being linked by consistency $(+)$ links D05+D06 and D05+H02. (Participants commonly use + to collect related data as well as for linking evidence to hypotheses.) After several pages concerning fading, they encounter a new page indicating that ALS-PD patients have high levels of aluminum in their brains. The transcript begins at this point. The subgraph that resulted from the interaction is shown in figure 4. (We added the labels on the boxes.)

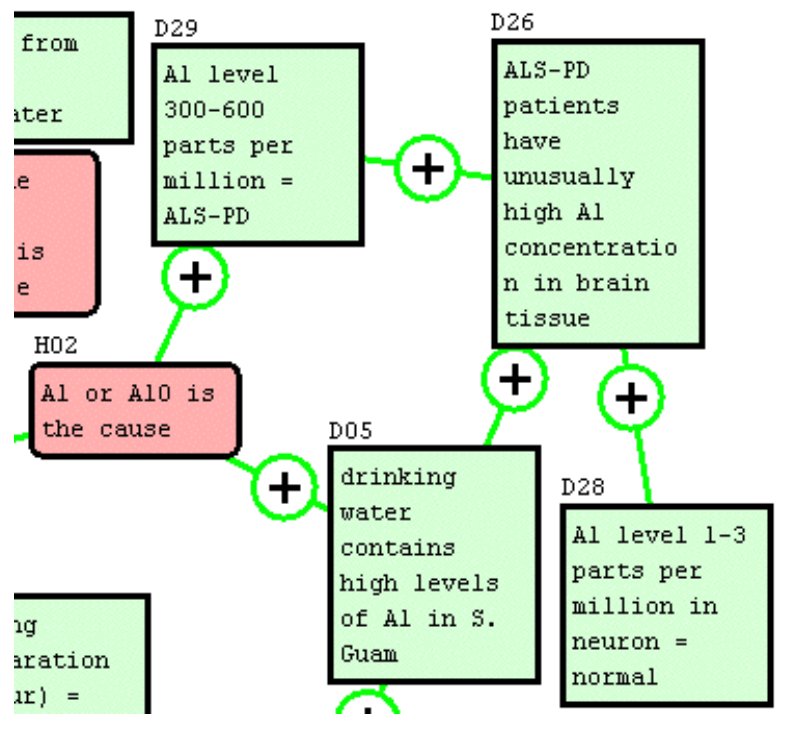

Figure 4. Graph fragment for example 1

At the information-uptake level of analysis, we see in figure 3 that there is information uptake through the graph in both directions: there are solid lines other than yellow with arrowheads going in both directions. Therefore, participants are collaborating through the graph; each is acting on information that was most recently provided or manipulated by the other. We can see that this interaction involves consistency links (green), deletion (maroon) and revision (blue); and that it draws upon material previously represented (lines going off the top of the image). This segment also exemplifies an asymmetric role division that was also seen in other pairs' sessions. P1 is taking responsibility for adding and editing the content of the text boxes, while P2 is linking together information contributed by both P1 and P2 (of four links, one involves only P1's material, two bring P1 and P2's material together, and one involves only P2's own material).

Stepping through this example, participants interacted as follows. P2 creates two data items D26 and D27 from the new information page. P1 is doing so at the same time, creating D28, which is redundant with D27. While P2 continues to work, P1 recognizes the redundancy, deletes P2's version (D28) and rewords his or her own version D27 to include some information from D28 (that it is about aluminum). Parallel redundant activity followed by merging and cleanup is common in our online transcripts.

Meanwhile, P2 goes on to add one more data item D29 and link it to D05. The manipulation of D05 is a reintroduction of an item that has not been considered for a while: this exemplifies the utility of a visual representation for reminding participants of previous information and enabling them to reference it easily. D05 was originally created and was last manipulated by P1; therefore this incident also illustrates one participant taking up information that had previously been contributed by another (as indicated by the solid line).

Almost a full minute after P1's deletion (they might have been absorbing what each other had just done), P2 links D26 to both his or her own D29 and P1's recent contribution D28, forming a cluster of related data. While P1 cleans up the wording of P2's recent contribution (D29), P2 now makes the evidential relationship to the aluminum hypothesis $\mathrm{H} 02$ explicit - again performing a reintroduction of an item originally introduced by P1. P2 now starts cleaning up in parallel to P1, by merging data items D05 and D06. After moving some things around to clean up the graph, participants finally acknowledge verbally their shared interpretation of they have achieved though the graph: "boy, we got something"; "heheh ALUMINUM!!!!"

It is clear that participants were collaborating through the graph, taking up information that was introduced by the other participant (evidence of grounding), and transforming joint representations to arrive at a conclusion (evidence of distributed cognition). Although the role distribution is asymmetric, the collaboration constitutes a form of knowledge construction in which they use the graph notation to come to agreement on the structure of evidence and its implication for a hypothesis under consideration. The verbal chat is only used to acknowledge what has been accomplished through the graph. Apparently, participants feel the need for a more explicit verbal form of grounding to mark the completion of their negotiated interpretation. 


\subsection{Example 2: Arguing Through the Graph}

The interaction of group 3 in example 2 exemplifies how a conversation-like interaction can take place through manipulation of the graph, and how conflict can be identified and addressed (albeit not satisfactorily in this case) via manipulations of the graph. The relevant actions are abstracted in the table of figure 5, where they are annotated with my conversational interpretation of those actions (thus, this column of the table differs from that in the previous example). To simplify the visual presentation, unrelated discussion was removed at the thick line in the graphic, and unrelated annotation links are removed for readability.

Examining the annotations we see that both participants (see arrowheads) are bearing the burden of collaboration by integrating each others' information (combinations of solid and dotted lines for three of the links). We also see that all polarities of evidential relations are being considered (grey, red, green). There is no chat in this segment: in fact, participants only chatted one more time, several pages later, on an unrelated hypothesis.

In this exchange, participants are exploring the implications of some new evidence for their second hypothesis (H02), that the cycad seeds cause the disease. Reading my interpretation down the right hand column of the table, this interaction has the form of a disagreement: P1 suggests (but then retracts) the possibility of a relationship (D13?H02); P2 proposes a negative relationship (H02-D13); then, after introducing some new data (D14), P1 proposes a positive relationship (D14+H02, where D14 is linked to D13), and deletes P2's proposed relationship. At the same time, P2 is using the new data P1 introduced to support his or her own interpretation (D14+D13). Participants are clearly engaging in a form of argumentation through the graph, without using the chat tool.

Upon closer examination the source of the disagreement can be seen to be an erroneous reading of the text. The text contains a double negative" "Such symptoms are not unlike those of someone with ALS." P2 apparently read this as simple negation, writing that the animals "didn't have the same symptoms as some one w/als." This error accounts for P2's confidence that the data conflicts with H02. Apparently, the participants did not identify the source of their disagreement in this error of interpretation.

This case exemplifies a situation in which the externalization of ideas led to identifications of differences of interpretation that were subsequently taken up by the individuals for attempted resolution via manipulations of the graph alone, at least for short episodes. Again, knowledge construction is possible through the graph. However, we must not neglect ways in which both notational and linguistic media are used in a coordinated manner for collaboration, as this tells us something about the limitations of the graph medium.

\subsection{Collaborating Through Chat and Graph}

With a few exceptions at the end of the sessions, most of the task-oriented interaction took place through the graph. However, chat at times played a crucial role in supporting the communication. There was also one session in which participants discussed what to do extensively in the chat.

The graph, naturally, was used primarily for what its representational primitives support: reporting and recording information gleaned from the source pages, proposing hypotheses, and indicating consistency and inconsistency relationships between these items. The graph was the primary means of accomplishing these communications, although there are a few examples of chat that could have been accomplished via the graph notation provided.

Some pairs used chat primarily for social banter as they carried out task-oriented interactions in the graph. Typically this social use of chat was occasionally punctuated with task-oriented chat, such as role assignments ("you do this one, OK?") and coordination of page turning ("ready?" "next?", etc.). Occasionally, brief chat exchanges during the session would focus on the value or interpretation of information, especially when a problematic situation arose. Often the interaction in such cases was multi-modal, involving use of both graph and chat.

An example is found in the portion omitted from the figure for Example 2. Participants discuss what to make of some new information that causes problems for one of their previous hypotheses. A brief verbal exchange is summarized by P1's action in the graph (Table 2). This kind of movement from verbal discussion to graph was typical of the conversations in face-to-face studies [17], but less typical online, where participants more typically generated a proposal in the graph and then discussed it [16].

Interestingly, several groups engaged in extended evaluative/interpretive discussions after reaching the final page, which announced that participants' "library research" was done. Analysis of these conversations to infer how they might be making use of the visual representation is on my future agenda. 


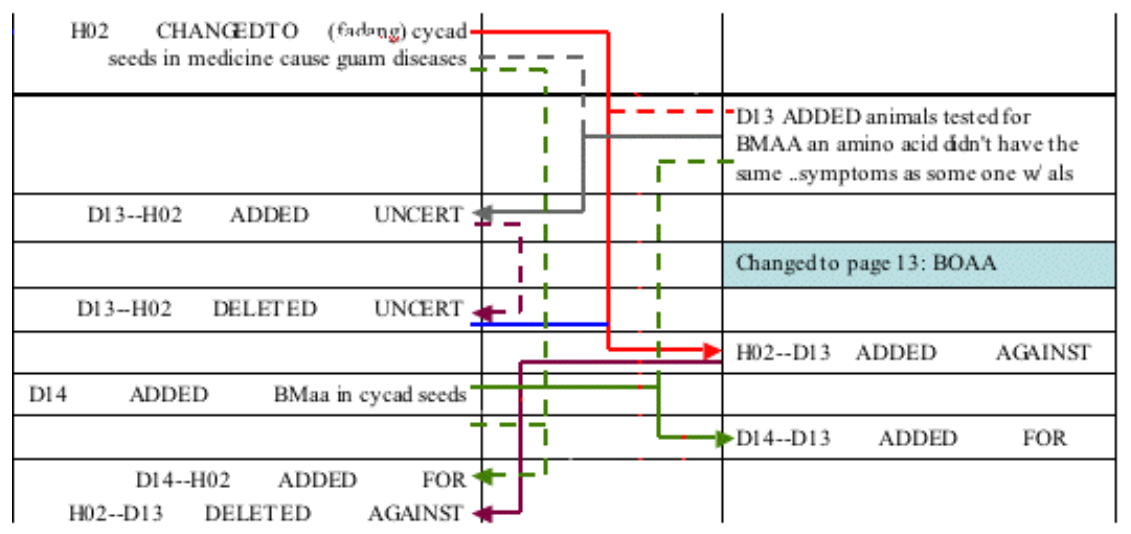

Context: Previously expressed by P1: H02 "(fading) cycad seeds in medicine cause guam diseases"

Participants have just read page 12 titled "BMAA-fed Monkeys Exhibit Signs of ALS-PD" reading "When scientists fed large doses of BMAA (an amino acid found in cycad seeds) to macaque monkeys, they observed the monkeys age before their eyes. After a few weeks' exposure to BMAA, some of the animals became weak. Over three months, some of the animals became apathetic, listless. Their hands trembled. They stooped and shuffled. Such symptoms are not unlike those of someone with ALS."

\begin{tabular}{lllll}
\hline Time & Who & Act & Object(s) & $\begin{array}{l}\text { Content (plaintext) or researcher's interpretation (italics) of act } \\
\text { animals tested for BMAA an amino acid didn't have the same ... symptoms as } \\
\text { some one w/ als }\end{array}$ \\
\hline $14: 35: 40$ & P2 & A & D13 & I think that has something to do with H02, but I'm not sure what. \\
\hline $14: 36: 10$ & P1 & A & D13?H02 & Never mind. \\
\hline 14:36:23 & & D & D13?H02 & They conflict. \\
\hline $14: 36: 28$ & P2 & A & H02-D13 & But it says that BMaa in cycad seeds \\
\hline $14: 36: 36$ & P1 & A & D14 & Right, that's why. \\
\hline $14: 36: 51$ & P2 & A & D14+D13 & So it's for the hypothesis. \\
\hline $14: 36: 51$ & P1 & A & D14+H02 & You're wrong. \\
\hline $14: 36: 56$ & & D & H02-D13 &
\end{tabular}

Figure 5: Arguing through the graph (researcher's interpretation)

\section{Table 2. Responding to action in graph}

\begin{tabular}{|c|c|c|c|}
\hline \multicolumn{4}{|c|}{$\begin{array}{l}\text { Context: Both participants have just opened a page titled } \\
\text { "Aluminum Abundant Throughout the World" reading } \\
\text { "Aluminum is the third-most abundant element on earth. People } \\
\text { are exposed to it all the time, in solid, dust, and food. According } \\
\text { to neuropathologist Daniel Perl, "the cause of the disease on } \\
\text { Guam is certainly not simple exposure to aluminum. I could } \\
\text { name fifty places all over the world that have aluminum-rich } \\
\text { soil."." }\end{array}$} \\
\hline Who & $A c t$ & Object(s) & Chat or graph content \\
\hline P1 & $\mathrm{C}$ & & so its not aluminum then? \\
\hline $\mathrm{P} 2$ & $\mathrm{C}$ & & $\begin{array}{l}\text { maybe it is? aluminum poision? } \\
\text { ing? i dunno? }\end{array}$ \\
\hline P1 & $\mathrm{C}$ & & maybe but never heard of that \\
\hline $\mathrm{P} 2$ & $\mathrm{C}$ & & ya \\
\hline \multirow[t]{2}{*}{ P1 } & A & D12 & aluminum poinsoning???? \\
\hline & A & D12!D08 & \\
\hline
\end{tabular}

\section{Conclusions}

Previous research by the author had studied external visual representations as supports for collaborative learning from a quantitative perspective, finding that the features of such representations can influence collaborative activity [17], and showing that representations are used differently online than face-toface, with more of the communicative function switching to visual representations online [16]. The present study was motivated by a desire to understand, from a qualitative perspective, exactly how shared external representations are used by participants to support their knowledge construction. A case-analysis is being undertaken on transcript segments in which participants acted intensively on the graph. Driven by two questions, we coded actions for "informational uptake" events, and then interpreted sequences of such events as knowledge construction episodes.

Preliminary responses to the questions can now be offered. First, it is possible to view manipulations of the diagrammatic representations as "conversational," in a manner similar to verbal interactions (participants are not merely operating in parallel in the same workspace). Example 1 showed how agreement can be reached through joint manipulation of a graphical medium, and Example 2 showed how argumentation is possible in such a medium. Second, interaction through the graph displays 
many of the criteria for knowledge construction (accretion of interpretations on an expanding knowledge base) suggested by theory, including grounding by implicit uptake of the interlocutor's actions in the graph, interactions that respond to and address differences of interpretation, and transformations of representations by multiple individuals leading to a joint solution.

The claim that knowledge construction can take place through the graph should be qualified. Although much of the interaction concerning hypotheses and evidence (i.e., those supported by our particular graph) take place through the graph, linguistic representations are used both as a back channel for social and task-coordination interactions, and are relied on when critical events occur requiring evaluation of the propositions that can be expressed in the graph. Future work should further clarify the roles of each medium of interaction as well as how to coordinate the two effectively. Also, interaction through a graph is different than language. Although this work demonstrated that not only grounding but also collaboration and knowledge construction is possible through visual as well as linguistic representations, there is no reason to believe that the structure of interactions through a graph are necessarily isomorphic to linguistic discourse. Future work includes raising the level of analysis further to identify recurring patterns of knowledge-constructive interactions that take place via the notational medium.

\section{References}

[1] Bertelsen, Olav W. and Bødker, Susanne (2003). Activity Theory. In J. M. Carroll (Ed.), HCI Models, Theories and Frameworks: Towards a Multidisiplinary Science. San Francisco, Mogan Kaufmann: 291-324.

[2] Blackwell, Alan \& Green, Thomas (2003). Notational Systems--The Cognitive Dimensions of Notations Framework. In J. M. Carroll (Ed.), HCI Models, Theories and Frameworks: Towards a Multidisiplinary Science. San Francisco, Mogan Kaufmann: 103-133.

[3] Clark, H.H. \& Brennan, S.E. (1991). Grounding in Communication. In L.B. Resnick, J.M. Levine and S.D. Teasley (eds.), Perspectives on Socially Shared Cognition (pp. 127-149). Hyattsville, MD: American Psychological Association.

[4] Collins, A. \& Ferguson, W. (1993). Epistemic forms and epistemic games: Structures and strategies to guide inquiry. Educational Psychologist, 28(1), 25-42.

[5] Doise, W., and Mugny, G. (1984) The Social Development of the Intellect, International Series in Experimental Social Psychology, vol. 10, Pergamon Press

[6] Festinger, L. (1957). A Theory of Cognitive Dissonance, Stanford University Press, 1957.

[7] Hollan, J., E. Hutchins, \& Kirsh, D.. (2002). Distributed Cognition: Toward a New Foundation for Human-Computer
Interaction Research. In J.M. Carroll (Ed.), Human-Computer Interaction in the New Millennium. New York, ACM Press Addison Wesley: 75-94.

[8] Hollan, J. \& Stornetta, S. (1992). Beyond being there. Proc. SIGCHI Conference on Human Factors in Computing Systems (CHI'92), Monterey, California, pp. 119-125.

[9] Monk, A. (2003). Common Ground in Electronically Mediated Communication: Clark's Theory of Language use. In J. M. Carroll (Ed.), HCI Models, Theories and Frameworks: Towards a Multidisiplinary Science. San Francisco, Mogan Kaufmann: 265-289.

[10] Mühlenbrock, M., \& Hoppe, U. (1999). Computer Supported Interaction Analysis of Group Problem Solving. Proc. Computer Support for Collaborative Learning Conference (CSCL '99), C. Hoadley \& J. Roschelle (Eds.) Dec. 12-15, Stanford University, Palo Alto, California. Mahwah, NJ: Lawrence Erlbaum Associates.

[11] Olson, G. M \& Olson, J. S. (2000). Distance Matters. In J. $\mathrm{M}$ Carroll (Ed) Human-Comuter Interaction in the New Millennum. New York: ACM Press (2002). pp. 397-417.

[12] Roschelle, J. (1994, May). Designing for cognitive communication: Epistemic fidelity or mediating collaborative inquiry? The Arachnet Electronic Journal of Virtual Culture, 2(2).

[13] Scardamalia, M. \& Bereiter, C. (2003). Knowledge building environments: Extending the limits of the possible in education and knowledge work. In A. DiStefano, K.E., Rudestam \& R. Silverman (Eds.), Encyclopedia of Distributed Learning. Thousand Oaks, CA: Sage Publications.

[14] Stahl, G. (2000). A model of collaborative knowledgebuilding. Proc. Fourth International Conference of the Learning Sciences (ICLS 2000), Ann Arbor, MI, pp. 70-77.

[15] Suthers, D, Connelly, J., Lesgold, A., Paolucci, M., Toth, E., Toth, J., and Weiner, A. (2001). Representational and Advisory Guidance for Students Learning Scientific Inquiry. In K. D. Forbus and P. J. Feltovich (Eds.) Smart Machines in Education: The Coming Revolution in Educational Technology (pp. 7-35). Menlo Park: AAAI Press.

[16] Suthers, D., Girardeau, L. and Hundhausen, C. (2003). Deictic Roles of External Representations in Face-to-face and Online Collaboration. Designing for Change in Networked Learning Environments, Proc. International Conference on Computer Support for Collaborative Learning, B. Wasson, S. Ludvigsen \& U. Hoppe (Eds), Dordrecht: Kluwer Academic Publishers, pp. 173-182.

[17] Suthers, D., and Hundhausen, C. (2003). An Empirical Study of the Effects of Representational Guidance on Collaborative Learning. Journal of the Learning Sciences, 12(2), 183-219.

This work was supported by the National Science Foundation under award 0093505. Any opinions, findings, and conclusions or recommendations expressed in this paper are those of the authors and do not necessarily reflect the views of the National Science Foundation. 